\title{
JOURNAL.RU
}

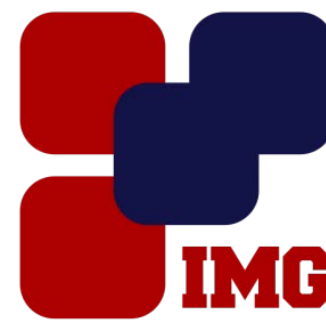
IVANOY Management Group

Макаров М.В. Казанский (Приволжсккий) федеральный университет Казанский национальный исследовательский технический университет им. А.Н. ТуполеваКАИ Казань, Россия

doi: 10.18411/lj-31-10-2016-2-07

idsp 000001:lj-31-10-2016-2-07

\section{О решении геометрически нелинейных и линейных задач о поперечном изгибе жестко закрепленной трехслойной пластины с трансверсально- мягким заполнителем}

\section{Введение.}

Интерес к композитным материалам вызван высоким уровнем их конструктивных свойств: прочности, жесткости и т. П. Для того, чтобы облегчить конструкцию, не уменьшив при этом ее несущую способность, используются тонкостенные элементы в виде оболочек. Такие оболочки широко распространены в инженерных сооружениях, машиностроении, судостроении, в авиационной промышленности и ракетной технике [1-5].
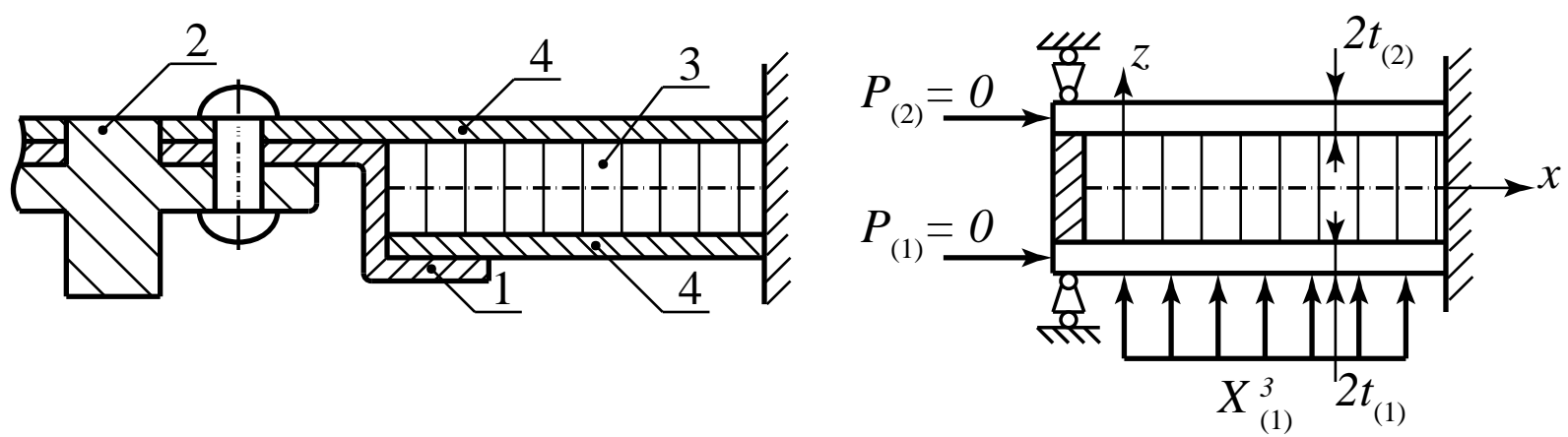

Рис. 1. Трехслойная пластина с трансверсально-мягким заполнителем (1 - подкрепляющая несущие слои диафрагма; 2 - жесткий опорный элемент; 3 - заполнитель; 4 - внешние несущие слои)

В настоящей работе изучаются геометрически линейная и нелинейная задачи о поперечном изгибе трехслойной пластины с трансверсально-мягким заполнителем (см. рис. 1a), схема нагружения и закрепления которой показана на рис. 1б. Построены конечно-разностные аппроксимации задач. Численная 
реализация разностной схемы в нелинейном случае осуществляется с помощью двухслойного итерационного метода. В среде Matlab разработаны комплексы программ для реализации приближенных методов, проведены численные эксперименты для модельных геометрически линейных и нелинейных задач. Проведен анализ полученных результатов. Ранее подобная задача изучалась в случае жесткого закрепления несущих слоев при отсутствии диафрагм [6-8]. В работах [9-16] изучены задачи об изгибе трехслойной пластины с трансверсально-мягким заполнителем в геометрически линейной и физически нелинейной постановке. Отметим, что физически нелинейные задачи теории мягких сетчатых оболочек исследованы в [17-25].

\section{1. Постановки задач.}

Изучаются задачи об изгибе трехслойной пластины с трансверсальномягким заполнителем. Для описания напряженно-деформированного состояния в несущих слоях используются уравнения модели Кирхгофа-Лява, в заполнителе - уравнения теории упругости, упрощенные в рамках принятой модели трансверсально-мягкого слоя и проинтегрированных по толщине с удовлетворением условий сопряжения слоев по перемещениям [26-28]. Ширина пластины равна $a$, толщина заполнителя составляет $2 \mathrm{t}$, толщины несущих слоев равны $2 t_{(k)}$, где $\mathrm{k}$ - номер слоя. Исследование процессов деформирования таких элементов диктуется прежде всего необходимостью определения степени их пригодности для дальнейшего использования. В соответствии с [26-28] введем обозначения: $H_{(k)}=t+t_{(k)}$ (здесь и всюду в дальнейшем предполагаем, что $\mathrm{k}=$ $1,2), \quad X_{(k)}^{1}, X_{(k)}^{3}$ - компоненты поверхностной нагрузки, приведенной к срединной поверхности k-го слоя, $w^{(k)}$ и $u^{(k)}-$ прогибы и осевые перемещения точек срединной поверхности k-го слоя соответственно, $q^{1}$ - касательные напряжения в заполнителе, $T_{(k)}^{11}, M_{(k)}^{11}$ - мембранные усилия и внутренние изгибающие моменты в k-м слое соответственно, $c_{3}=E_{3} /(2 h), M_{(k)}^{1}$ компоненты вектора поверхностных моментов $k$-го слоя.. $M_{(k)}^{11}=D_{(k)} d^{2} u^{(k)} / d x^{2}$, где $D_{(k)}=B_{(k)} h_{(k)}^{2} / 3$ - изгибная жесткость к-го 
слоя, $B_{(k)}=2 h_{(k)} E^{(k)} /\left(1-v_{12}^{(k)}\right)\left(1-v_{21}^{(k)}\right)$ - жесткость k-го слоя на растяжениесжатие, $E^{(k)}$ и $v_{12}^{(k)}, v_{21}^{(k)}$ - модуль упругости первого рода и коэффициенты Пуассона материала k-го несущего слоя, $S_{(k)}^{1}=Q_{(k)}^{1}+H_{(k)} q^{1}$ - обобщенные перерезывающие силы в $k_{\text {-м слое, }} Q_{(k)}^{1}=d M_{(k)}^{11} / d x+T_{(k)}^{11} d w^{(k)} / d x+M_{(k)}^{1}$

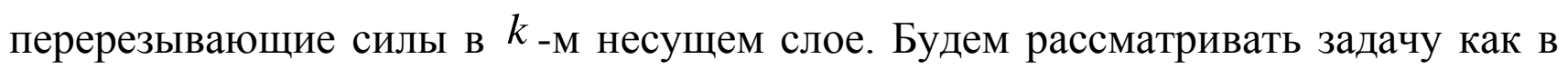
геометрически нелинейной постановке:

$$
T_{(k)}^{11}=B_{(k)}\left(d u^{(k)} / d x+\frac{1}{2}\left(d w^{(k)} / d x\right)^{2}\right),
$$

так и в геометрически линейной постановке:

$$
T_{(k)}^{11}=B_{(k)} d u^{(k)} / d x .
$$

Задача о равновесии трехслойной пластины с трансверсально-мягким заполнителем в одномерной геометрически нелинейной постановке описывается следующей системой дифференциальных уравнений

$$
\left\{\begin{array}{l}
\frac{d T_{(k)}^{11}}{d x}+X_{(k)}^{1}+(3-2 k) q^{1}=0, \quad 0<x<a, \\
\frac{d S_{(k)}^{1}}{d x}+c_{3}(3-2 k)\left(w^{(2)}-w^{(1)}\right)+X_{(k)}^{3}=0, \quad 0<x<a, \\
u^{(1)}-u^{(2)}-H_{(1)} \frac{d w^{(1)}}{d x}-H_{(2)} \frac{d w^{(2)}}{d x}+\frac{2 t}{G_{13}} q^{1}-\frac{2 t^{3}}{3 E_{3}} \frac{d^{2} q^{1}}{d x^{2}}=0,0<x<a,
\end{array}\right.
$$

Предполагаем, что в правом торцевом сечении края несущих слоев жестко защемлены и отсутствует адгезионное соединение заполнителя с опорным элементом, на левом торцевом сечении края несущих слоев пластины шарнирно оперты на абсолютно жесткие в поперечном направлении диафрагмы, склеенной с торцевым сечением заполнителя:

$$
\begin{aligned}
& T_{(k)}^{11}(0)=0, w^{(k)}(0)=d^{2} w^{(k)}(0) / d x^{2}=0, k=1,2, d q^{1}(0) / d x=0, \\
& u^{(k)}(a)=0, w^{(k)}(a)=d w^{(k)}(a) / d x=0, k=1,2, q^{1}(a)=0 .
\end{aligned}
$$


Пусть $U=\left(w^{(1)}, w^{(2)}, u^{(1)} w^{(2)}\right)$ - вектор перемещений точек срединной поверхности k-го слоя. Обобщенные постановки задач (1), (3)-(5) и (2)-(5) формулируется в виде интегральных тождеств относительно функций $U{ }_{\text {и }} q^{1}$ из пространств Соболева.

2. Приближенные методы решения и численные эксперименты.

Приближенные методы решения изучаемых задач основаны на их конечно-разностной аппроксимациях, в нелинейном случае для ее реализации используется двухслойный итерационный процесс, при этом разностная схема имеет вид $\left(A_{1}+A_{2}\right) U=F, A_{1}$ - линейный оператор, $A_{2}$ - нелинейный $[6,7]$. Двухслойный итерационный процесс ([6, 7, 29-34], см. также [35-40]) имеет вид $A_{1}\left(U^{(n+1)}-U^{(n)}\right) / \tau+\left(A_{1}+A_{2}\right) U^{(n)}=F, U^{(0)}$ - начальное приближение, $\tau>0$ итерационный параметр. В линейном случае проводится непосредственное решение соответствующих систем линейных алгебраических уравнений. Для модельных задач проведены численные эксперименты при следующих значениях параметров: $a=20 \mathrm{~cm}, t_{1}=t_{2}=0.05 \mathrm{~cm}, t=1 \mathrm{~cm}, G_{13}=25$ МПа, $E_{3}=50$ МПа, $\quad X_{(2)}^{3}=0, \quad E^{(k)}=133 \cdot 10^{3}$ МПа, $\quad v_{12}^{(k)}=v_{21}^{(k)}=0.3, \quad X_{(k)}^{1}=0$, $M_{(k)}^{1}=0, k=1,2, U^{(0)}=0$. Число точек сетки $N=500$. Поперечная нагрузка полагалась равной $X_{(1)}^{3}=0.1$ МПа (рис.2, 3, 6) и $X_{(1)}^{3}=1$ МПа (рис. 4, 5, 7). При малых нагрузках нелинейность проявляется очень слабо (осевые перемещения в линейном и нелинейном случаях почти совпали).

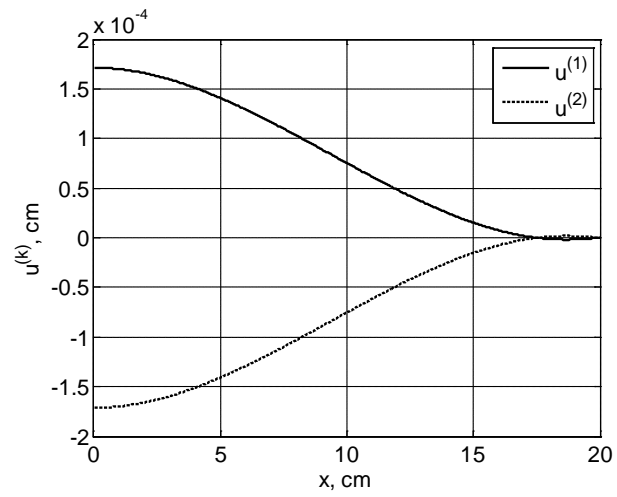

Рис. 2. Осевые перемещения линейный случай, $X_{(1)}^{3}=0.1$ МПа

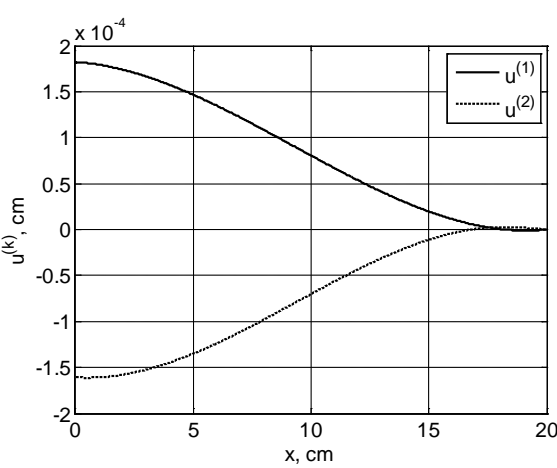

Рис. 3. Осевые перемещения

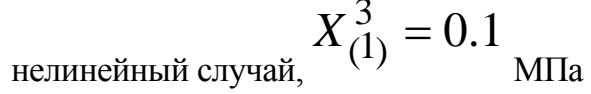




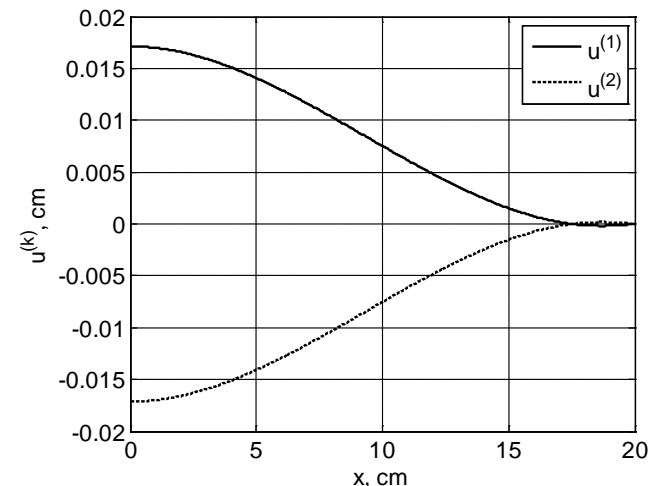

Рис. 4. Осевые перемещения,

$$
\text { линейный случай, }{ }_{(1)}^{3}=1_{\text {МПа }}
$$

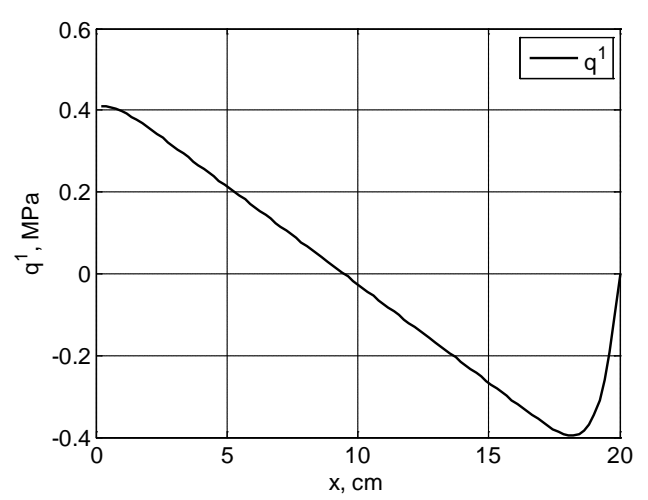

Рис. 6. Касательные напряжения в заполнителе,

$$
X_{(1)}^{3}=0.1_{\text {МПа }}
$$

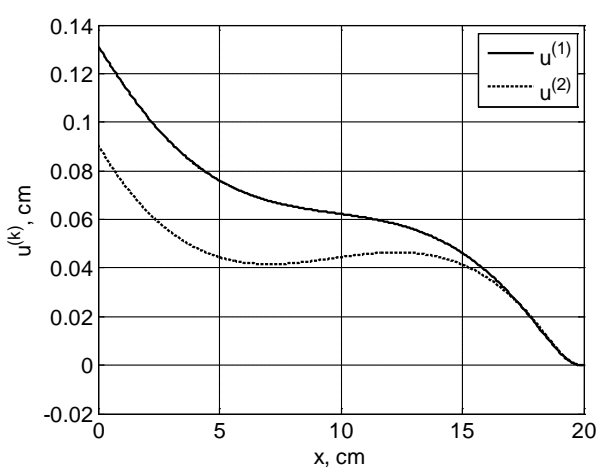

Рис. 5. Осевые перемещения

$$
\text { нелинейный случай } X_{(1)}^{3}=1_{\text {МПа }}
$$

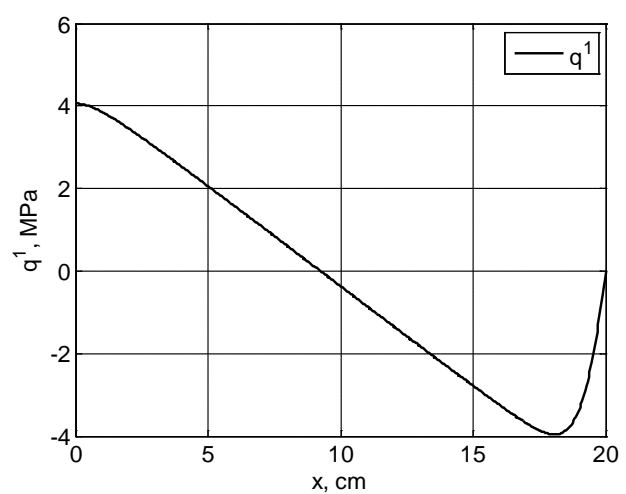

Рис. 7. Касательные напряжения в

$$
\text { заполнителе, } X_{(1)}^{3}=1_{\text {МПа }}
$$

При больших значениях нагрузки сказывается нелинейность - появляются значительные прогибы, поэтому перемещения каждой точки несущего слоя, который подвергнут растяжению и ещё изгибается, что и характеризует его перемещения с положительным знаком. Остальные характеристики практически совпадают, независимо от величины нагрузки (на рис. 6, 7 приведены значения касательных напряжений в заполнителе).

\section{3. Заключение.}

Проведено численное исследование геометрически линейной и нелинейной задач $о$ поперечном изгибе трехслойной пластины $c$ трансверсально-мягким заполнителем. Это позволит в дальнейшем исследовать формы потери устойчивости пластины [41-45] на основе метода продолжения по параметру. Исследование корректности постановки можно провести по аналогии с [46-53].

Публикация осуществлена при финансовой поддержке РФФИ (проекты 15-01-05686, 16-08- 



\section{Литература}

1. Коршаков А.В. Обзор некоторых методов реконструкции томографических изображений. //Контроль. Диагностика. - М.: Машиностроение.-№3(105) 2007.-с. 45-49

2. Бейтс Р., Мак-Доннелл М. Восстановление и реконструкция изображений: Пер. с англ. - М.: Мир, 1989. - 336 с.

3. Чичигин Б.А., Кеткович А.А., Чернов Л.А. Современные лазерные системы контроля геометрии сложных поверхностей //Тезисы докладов семнадцатой российской НТК с международным участием «Неразрушающий контроль и диагностка», Екатеринбург, ИМАШ, УрО PAH, 2005-c. 168.

4. Будадин О.Н., Бекаревич А.А., Морозова Т.Ю. Математическое моделирование процесса теплового контроля локальных дефектов металлических изделий сложной формы на примере лопаток турбин газотрубных двигателей В мире научных открытий. №8(68), Красноярск, 2015 , с. $34-43$.

5. Будадин О.Н., Бекаревич А.А., Морозова Т.Ю. Тепловой автоматизированный контроль качества и диагностики технического состояния лопаток турбин газотурбинных агрегатов с оценкой надежности эксплуатации. М.: Спектр, 2014, 382 с. 